\title{
Reliable Multi-GNSS Real-Time Kinematic Positioning
}

\author{
Michael Heinrich $^{1}$, Andreas Sperl ${ }^{1}$, Ulrich Mittmann ${ }^{1}$ and Patrick Henkel ${ }^{1}$ \\ 1 ANavS GmbH \\ 80798 Munich, Germany \\ \{michael.heinrich, andreas.sperl,ulrich.mittmann, patrick.henkel\}@anavs.de
}

\begin{abstract}
Surveying, agriculture and the navigation of autonomous robots, cars, ships and aerial systems require Global Navigation Satellite Systems (GNSS) for precise positioning.

In this paper, we describe a Real-Time Kinematic (RTK) positioning method, that uses both GPS and Galileo measurements with a common reference satellite, estimates a pseudorange multipath error for each satellite to prevent a mapping of multipath errors into the position, and performs a hypothesis testing for ambiguity fixing. The proposed method is tested on the new Multi-Sensor, Multi-GNSS RTK positioning module of ANavS, which includes up to 3 GNSS receivers, an inertial sensor, a barometer and a processor for RTK positioning on a single board. The measurement results show a repeatable millimeterlevel positioning accuracy.
\end{abstract}

Keywords-Multi-GNSS; Reliable RTK; LTE-interference

\section{INTRODUCTION}

Precise positioning is required for a variety of static and kinematic applications, e.g. for surveying, for agriculture and for navigation of autonomous robots, cars, ships and aerial systems. Real-Time Kinematic (RTK) positioning with a Global Navigation Satellite System (GNSS) has become increasingly popular over the last years since (a) it provides millimeter- to centimeter-level positioning accuracies, (b) GNSS signals are globally available and (c) GNSS receivers are quite cheap.

However, there are a few remaining challenges: First, pseudorange multipath, cycle slips and temporary losses of lock occur frequently in urban environments and reduce the positioning accuracy. Second, the fixing time of single-frequency RTK systems is still in the order of minutes. A third challenge is related to the periodicity of the ambiguous carrier phase measurements: The conventional integer fixing techniques (e.g. bootstrapping and the LAMBDA method of Teunissen [1]) are invariant with respect to errors in the float ambiguity solution. Thereby, any integer error in the float ambiguity estimates translates into a substantial positioning error.

This paper has the following objectives: We increase the number of available measurements by using both GPS and Galileo satellites and a common reference satellite for both constellations. Thereby, the fixing times are reduced. A pseudorange multipath parameter is estimated for each double difference pseudorange measurement as described by Henkel et al. in [2]. The ambiguity fixing of LAMBDA is enhanced by hypothesis testing, i.e. the accumulated sums of squared phase measurement residuals are tracked for multiple integer candidate vectors and used for final candidate selection. This paper also includes the presentation of a new RTK positioning module that includes up to 3 multi-constellation GNSS receivers (for attitude determination), an inertial sensor and a processor for implementing the proposed RTK positioning method and a tightly coupled sensor fusion on a single board.

The measurement results show a repeatable millimeter-level positioning accuracy with this new module.

\section{Multi-GNSS Real-Time Kinematic Positioning}

In this section, we describe our approach for Real-Time Kinematic (RTK) positioning using pseudorange and carrier phase measurements of both GPS and Galileo. The pseudorange measurement of satellite $k$ from the Global Navigation Satellite System (GNSS) $s \in\{1,2\}$ as provided by the Delay Locked Loop (DLL) of the $r$-th GNSS receiver is modeled according to Misra and Enge [3] as

$$
\begin{aligned}
\rho_{r}^{k, s}= & \left(\vec{e}_{r}^{k, s}\right)^{\mathrm{T}}\left(\vec{x}_{r}-\vec{x}^{k, s}\right)+c\left(\delta \tau_{r}^{s}-\delta \tau^{k, s}\right) \\
& +I_{r}^{k, s}+T_{r}^{k, s}+\Delta \rho_{\mathrm{MP}_{r}}^{k, s}+b_{r}+b^{k, s}+\eta_{r}^{k, s},
\end{aligned}
$$

with the direction vector $\vec{e}_{r}^{k, s}=\left(\vec{x}_{r}-\vec{x}^{k, s}\right) /\left\|\vec{x}_{r}-\vec{x}^{k, s}\right\|$ between satellite and receiver, the receiver position $\vec{x}_{r}$, the satellite position $\vec{x}^{k, s}$, the speed of light $c$ in vacuum, the receiver clock offset $\delta \tau_{r}^{s}$ being constellation-dependent (see description of GPS-Galileo Time Offset (GGTO) as described by Hahn and Powers in [4]), the satellite clock offset $\delta \tau^{k, s}$, the ionospheric delay $I_{r}^{k, s}$, the tropospheric delay $T_{r}^{k, s}$, the pseudorange multipath error $\Delta \rho_{\mathrm{MP}_{r}}^{k, s}$, the receiver code bias $b_{r}$, the satellite code bias $b^{k, s}$ and the receiver noise $\eta_{r}^{k, s}$.

The satellite position and clock offset is provided by the satellite navigation message. Thus, the respective terms can be accounted for, and corrected measurements are defined based on Eq. (1) as:

$$
\begin{aligned}
\tilde{\rho}_{r}^{k, s} & :=\rho_{r}^{k, s}+\left(\vec{e}_{r}^{k, s}\right)^{\mathrm{T}} \vec{x}^{k, s}+c \delta \tau^{k, s} \\
& =\left(\vec{e}_{r}^{k, s}\right)^{\mathrm{T}} \vec{x}_{r}+c \delta \tau_{r}^{s}+\tilde{\eta}_{r}^{k, s},
\end{aligned}
$$

with $\tilde{\eta}_{r}^{k, s}$ being the lumped sum of atmospheric errors, receiver and satellite biases, multipath errors and measurement noise:

$$
\tilde{\eta}_{r}^{k, s}:=I_{r}^{k, s}+T_{r}^{k, s}+\Delta \rho_{\mathrm{MP}_{r}}^{k, s}+b_{r}+b^{k, s}+\eta_{r}^{k, s} .
$$

The position determination requires the joint use of multiple satellites. Let us assume that $s=1$ refers to GPS with $K_{1}$ visible satellites and $s=2$ refers to Galileo with $K_{2}$ visible 
satellites. We stack the corrected pseudorange measurements of Eq. (2) of both GPS and Galileo in a single vector, i.e.

$$
\underbrace{\left(\begin{array}{c}
\tilde{\rho}_{r}^{1,1} \\
\vdots \\
\tilde{\rho}_{r}^{K_{1}, 1} \\
\tilde{\rho}_{r}^{1,2} \\
\vdots \\
\tilde{\rho}_{r}^{K_{2}, 2}
\end{array}\right)}_{\tilde{\rho}_{r}}=H_{r}\left(\begin{array}{c}
\vec{x}_{r} \\
c \delta \tau_{r}^{1} \\
c \delta \tau_{r}^{2}
\end{array}\right)+\underbrace{\left(\begin{array}{c}
\tilde{\eta}_{r}^{1,1} \\
\vdots \\
\tilde{\eta}_{r}^{K_{1}, 1} \\
\tilde{\eta}_{r}^{1,2} \\
\vdots \\
\tilde{\eta}_{r}^{K_{2}, 2}
\end{array}\right)}_{\tilde{\eta}_{r}},
$$

with

$$
H_{r}=\left(\begin{array}{ccc}
\left(\vec{e}_{r}^{1,1}\right)^{\mathrm{T}} & 1 & 0 \\
\vdots & \vdots & \vdots \\
\left(\vec{e}_{r}^{K_{1}, 1}\right)^{\mathrm{T}} & 1 & 0 \\
\hline\left(\vec{e}_{r}^{1,2}\right)^{\mathrm{T}} & 0 & 1 \\
\vdots & \vdots & \vdots \\
\left(\vec{e}_{r}^{K_{2}, 2}\right)^{\mathrm{T}} & 0 & 1
\end{array}\right) .
$$

The absolute receiver position and clock offsets are obtained by minimizing the sum of squared residuals, i.e.

$$
\begin{aligned}
\left(\begin{array}{c}
\hat{\vec{x}}_{r} \\
c \delta \hat{\tau}_{r}^{1} \\
c \delta \hat{\tau}_{r}^{2}
\end{array}\right) & =\left(\begin{array}{c}
\min _{\vec{x}_{r}} \\
c \delta \tau_{r}^{1} \\
c \delta \tau_{r}^{2}
\end{array}\right)\left\|\tilde{\rho}_{r}-H_{r}\left(\begin{array}{c}
\vec{x}_{r} \\
c \delta \tau_{r}^{1} \\
c \delta \tau_{r}^{2}
\end{array}\right)\right\|_{\Sigma_{\tilde{\rho}_{r}-1}^{2}}^{2} \\
& =\left(H_{r}^{\mathrm{T}} \Sigma_{\tilde{\rho}_{r}}^{-1} H_{r}\right)^{-1} H_{r}^{\mathrm{T}} \Sigma_{\tilde{\rho}_{r}}^{-1} \tilde{\rho}_{r} .
\end{aligned}
$$

The accuracy of the absolute positioning is limited by atmospheric errors and pseudorange multipath, and typically in the order of a few meters.

A higher positioning accuracy is obtained by differential positioning between the user (being indexed by $u$ ) and a closeby reference station (being indexed by $r$ ) since the common atmospheric errors, residual orbital and satellite clock errors and biases are eliminated by differencing the measurements between the user and the reference station. The positioning accuracy can be further improved by using the much more accurate carrier phase measurements.

We select a common reference satellite for all visible satellites of both constellations and denote it by $l, \bar{s}$. The double difference measurement between the receiver pair $u$ and $r$ and the satellite pair $k, s$ and $l, \bar{s}$ is modeled according to Henkel et al. [2] as

$$
\begin{aligned}
\rho_{u r}^{k, s ; l, \bar{s}}:= & \left(\rho_{u}^{k, s}-\rho_{r}^{k, s}\right)-\left(\rho_{u}^{l, \bar{s}}-\rho_{r}^{l, \bar{s}}\right) \\
= & \left(\left(\vec{e}_{u}^{k, s}\right)^{\mathrm{T}}\left(\vec{x}_{u}-\vec{x}^{k, s}\right)-\left(\vec{e}_{r}^{k, s}\right)^{\mathrm{T}}\left(\vec{x}_{r}-\vec{x}^{k, s}\right)\right) \\
& -\left(\left(\vec{e}_{u}^{l, \bar{s}}\right)^{\mathrm{T}}\left(\vec{x}_{u}-\vec{x}^{l, \bar{s}}\right)-\left(\vec{e}_{r}^{l, \bar{s}}\right)^{\mathrm{T}}\left(\vec{x}_{r}-\vec{x}^{l, \bar{s}}\right)\right) \\
& +\left(c \delta \tau_{u}^{s}-c \delta \tau_{r}^{s}\right)-\left(c \delta \tau_{u}^{\bar{s}}-c \delta \tau_{r}^{\bar{s}}\right) \\
& +\Delta \rho_{\mathrm{MP}_{u r}}^{k, s ; l, \bar{s}}+\eta_{u r}^{k, s ; l, \bar{s}} .
\end{aligned}
$$

We use the approximation that the GGTO (GPS-Galileo Time Offset) is equal for both receivers [4], i.e.

$$
\delta \tau_{u}^{s}-\delta \tau_{u}^{\bar{s}} \approx \delta \tau_{r}^{s}-\delta \tau_{r}^{\bar{s}} \quad \forall s \neq \bar{s}
$$

to approximate Eq. (7) by:

$$
\begin{aligned}
\rho_{u r}^{k, s ; l, \bar{s}} \approx & \left(\left(\vec{e}_{u}^{k, s}\right)^{\mathrm{T}}\left(\vec{x}_{u}-\vec{x}^{k, s}\right)-\left(\vec{e}_{r}^{k, s}\right)^{\mathrm{T}}\left(\vec{x}_{r}-\vec{x}^{k, s}\right)\right) \\
& -\left(\left(\vec{e}_{u}^{l, \bar{s}}\right)^{\mathrm{T}}\left(\vec{x}_{u}-\vec{x}^{l, \bar{s}}\right)-\left(\vec{e}_{r}^{l, \bar{s}^{\mathrm{T}}}\right)^{\mathrm{T}}\left(\vec{x}_{r}-\vec{x}^{l, \bar{s}^{\prime}}\right)\right) \\
& +\Delta \rho_{\mathrm{MP}_{u r}^{k, s ; l, \bar{s}}}^{k, \eta_{u r}^{k, s ; l, \bar{s}}}
\end{aligned}
$$

The double difference pseudorange measurements of Eq. (7) are corrected for the known satellite positions of both GNSS constellations and the known position $\vec{x}_{r}$ of the reference station, i.e.

$$
\begin{aligned}
\tilde{\rho}_{u r}^{k, s ; l, \bar{s}}:= & \rho_{u r}^{k, s ; l, \bar{s}} \\
& -\left(\left(\vec{e}_{u}^{k, s}\right)^{\mathrm{T}}\left(\vec{x}_{r}-\vec{x}^{k, s}\right)-\left(\vec{e}_{r}^{k, s}\right)^{\mathrm{T}}\left(\vec{x}_{r}-\vec{x}^{k, s}\right)\right) \\
& +\left(\left(\vec{e}_{u}^{l, \bar{s}}\right)^{\mathrm{T}}\left(\vec{x}_{r}-\vec{x}^{l, \bar{s}}\right)-\left(\vec{e}_{r}^{l, \bar{s}}\right)^{\mathrm{T}}\left(\vec{x}_{r}-\vec{x}^{l, \bar{s}}\right)\right) .
\end{aligned}
$$

Replacing $\rho_{u r}^{k, s ; l, \bar{s}}$ in Eq. (10) by Eq. (7) yields

$$
\begin{aligned}
\tilde{\rho}_{u r}^{k, s ; l, \bar{s}}= & \left(\vec{e}_{u}^{k, s}-\vec{e}_{u}^{l, \bar{s}}\right)^{\mathrm{T}}\left(\vec{x}_{u}-\vec{x}_{r}\right) \\
& +\Delta \rho_{\mathrm{MP}_{u r}^{k, s ; l}}+\eta_{u r}^{k, s ; l, \bar{s}},
\end{aligned}
$$

where the direction vectors are known with sufficient accuracy from the absolute positioning, and $\vec{x}_{u}-\vec{x}_{r}$ denotes the unknown relative position ("baseline") between both receivers.

The (corrected) double difference carrier phase measurements are modeled similar to the double difference pseudorange measurements, i.e.

$$
\lambda \tilde{\varphi}_{u r}^{k, c ; l, \bar{s}}=\left(\vec{e}_{u}^{k, s}-\vec{e}_{u}^{l, \bar{s}}\right)^{\mathrm{T}}\left(\vec{x}_{u}-\vec{x}_{r}\right)+\lambda N_{u r}^{k, s ; l, \bar{s}}+\varepsilon_{u r}^{k, s ; l, \bar{s}},
$$

with wavelength $\lambda$, integer ambiguity $N_{u r}^{k, c ; l, \bar{s}} \in \mathbb{Z}$ due to the periodicity of the carrier phase measurements, and double difference phase noise $\varepsilon_{u r}^{k, s ; l, \bar{s}}$ including phase multipath. The corrected double differenced pseudorange and carrier phase measurements of both constellations are stacked in a single measurement vector at epoch $n$, i.e.

$$
\begin{aligned}
z_{n}=\quad & \left(\tilde{\rho}_{u r}^{1,1 ; l, \bar{s}}, \ldots, \tilde{\rho}_{u r}^{1, K_{1} ; l, \bar{s}}, \tilde{\rho}_{u r}^{2,1 ; l, \bar{s}}, \tilde{\rho}_{u r}^{2, K_{2} ; l, \bar{s}}, \ldots\right. \\
& \left.\tilde{\varphi}_{u r}^{1,1 ; l, \bar{s}}, \ldots, \tilde{\varphi}_{u r}^{1, K_{1} ; l, \bar{s}}, \tilde{\varphi}_{u r}^{2,1 ; l, \bar{s}}, \ldots, \tilde{\varphi}_{u r}^{2, K_{2} ; l, \bar{s}}\right)^{\mathrm{T}} .
\end{aligned}
$$

This measurement vector can be expressed in terms of the unknowns as given by Eq. (11) and (12), i.e.

$$
z_{n}=H_{u, n} \xi_{u r, n}+A N_{u r}+\eta_{z_{n}}
$$

with

$$
H_{u, n}=\left(\begin{array}{c|cc}
H_{u, n}^{1} & I^{K_{1} \times K_{1}} & 0^{K_{1} \times K_{2}} \\
H_{u, n}^{2} & 0^{K_{2} \times K_{1}} & I^{K_{2} \times K_{2}} \\
\hline H_{u, n}^{1} & 0^{K_{1} \times K_{1}} & 0^{K_{1} \times K_{2}} \\
H_{u, n}^{2} & 0^{K_{2} \times K_{1}} & 0^{K_{2} \times K_{2}}
\end{array}\right),
$$


in which the matrices $H_{u, n}^{1}$ and $H_{u, n}^{2}$ are defined as

$$
H_{u, n}^{1}=\left(\begin{array}{c}
\left(\vec{e}_{u}^{1,1}-\vec{e}_{u}^{l, \bar{s}}\right)^{\mathrm{T}} \\
\vdots \\
\left(\vec{e}_{u}^{K_{1}, 1}-\vec{e}_{u}^{l, \bar{s}}\right)^{\mathrm{T}}
\end{array}\right)
$$

and

$$
H_{u, n}^{2}=\left(\begin{array}{c}
\left(\vec{e}_{u}^{1,2}-\vec{e}_{u}^{l, \bar{s}}\right)^{\mathrm{T}} \\
\vdots \\
\left(\vec{e}_{u}^{K_{2}, 2}-\vec{e}_{u}^{l, \bar{s}}\right)^{\mathrm{T}}
\end{array}\right),
$$

and $\xi_{u r, n}$ includes all real-valued unknowns, i.e.

$$
\begin{aligned}
\xi_{u r, n}= & \left(\left(\vec{x}_{u}-\vec{x}_{r}\right)^{\mathrm{T}}, \Delta \rho_{\mathrm{MP}_{u r}}^{1,1 ; l, \bar{s}}, \ldots, \Delta \rho_{\mathrm{MP}_{u r}, 1 ; l, \bar{s}}^{K_{1}}, \ldots\right. \\
& \Delta \rho_{\mathrm{MP}_{u r}, 2 ; l, \bar{s}}^{\left.1, \ldots, \Delta \rho_{\mathrm{MP}_{u r}}^{K_{2}, 2 ; l, \bar{s}}\right)^{\mathrm{T}} .}
\end{aligned}
$$

The matrix $A$ describes the mapping of the ambiguities into the measurement space, i.e.

$$
A=\left(\begin{array}{r}
0^{\left(K_{1}+K_{2}\right) \times\left(K_{1}+K_{2}\right)} \\
\lambda \cdot I^{\left(K_{1}+K_{2}\right) \times\left(K_{1}+K_{2}\right)}
\end{array}\right),
$$

and the vector $N_{u r}$ includes all double difference integer ambiguities, i.e.

$N_{u r}=\left(N_{u r}^{1,1 ; l, \bar{s}}, \ldots, N_{u r}^{K_{1}, 1 ; l, \bar{s}}, N_{u r}^{1,2 ; l, \bar{s}}, \ldots, N_{u r}^{K_{2}, 2 ; l, \bar{s}}\right)^{\mathrm{T}} \in \mathbb{Z}$,

and $\eta_{z_{n}}$ includes the stacked pseudorange and carrier phase noise. The number of unknown position coordinates, carrier phase ambiguities and pseudorange multipath errors exceeds the number of measurements for a single epoch. Therefore, the measurements of multiple epochs need to be considered in combination with the time-constant ambiguities.

A state space model describes the temporal evolution of the state parameters and, thereby, strengthens the system model. The state space model is given by Henkel et al. [2] as

$$
\xi_{u r, n}=\Phi \xi_{u r, n-1}+\eta_{\xi_{u r, n}}
$$

with the state transition matrix $\Phi$, the state vector $\xi_{u r, n-1}$ of the previous epoch and the process noise $\eta_{\xi_{u r, n}}$. The state transition matrix becomes an identity matrix for static positioning, i.e.

$$
\Phi=1^{\left(3+K_{1}+K_{2}\right) \times\left(3+K_{1}+K_{2}\right)} .
$$

We use a standard Kalman filter (see Brown and Hwang [5]) to estimate the state parameters from the measurements using both measurement and state space models. The obtained ambiguity estimate of the Kalman filter is denoted by $\hat{N}_{u r}^{+}$and is typically a real-valued vector since the Kalman filter does not consider the integer property of the ambiguities.

Therefore, we search for all integer candidate vectors $N_{u r}^{j}$ within a certain search space $\chi^{2}$ around the float solution, i.e

$$
\left\|\hat{N}_{u r}^{+}-N_{u r}^{j}\right\|_{\Sigma_{\hat{N}_{u r}}^{-1}}^{2} \leq \chi^{2},
$$

where $\Sigma_{\hat{N}_{u r}}$ denotes the float ambiguity covariance matrix. The ambiguity residuals $\hat{N}_{u r}^{+}-N_{u r}^{j}$ are invariant with respect to integer errors of the float solution and, therefore, are not appropriate for validation of the ambiguity fixing.

Therefore, a hypothesis testing is performed, i.e. the sum of squared measurement residuals is determined for each candidate vector and epoch $n$, and accumulated over time. Finally, the candidate vector that minimizes the accumulated sum of squared measurement residuals is considered to be most trustworthy, i.e.

$$
\check{N}_{u r}=\arg \min _{N_{u r}^{j}}\left(\sum_{n=1}^{N} \min _{\xi_{u r, n}}\left\|z_{n}-H_{u, n} \xi_{u r, n}-A N_{u r}^{j}\right\|_{\Sigma_{z_{n}}^{-1}}^{2}\right),
$$

where $\Sigma_{z_{n}}$ denotes the measurement covariance matrix. Once the ambiguities are fixed, the fixed baseline and multipath errors are obtained by least-squares estimation, i.e.

$$
\begin{aligned}
\check{\xi}_{u r, n} & =\arg \min _{\xi_{u r, n}}\left\|z_{n}-H_{u, n} \xi_{u r, n}-A \check{N}_{u r}\right\|_{\Sigma_{z_{n}}^{-1}}^{2} \\
& =\left(H_{u, n}^{\mathrm{T}} \Sigma_{z_{n}}^{-1} H_{u, n}\right)^{-1} H_{u, n}^{\mathrm{T}} \Sigma_{z_{n}}^{-1}\left(z_{n}-A \check{N}_{u r}\right) .
\end{aligned}
$$

\section{DESCRIPTION OF THE NOVEl ANAVS Multi-SENSOR, Multi-GNSS RTK Positioning Module}

The proposed method of the previous section was implemented on the novel Multi-Sensor, Multi-GNSS RTK positioning module of ANavS. The module has the following features:

- 3 integrated GNSS receivers with external antennas for precise attitude determination

- 1 integrated inertial measurement unit with 3D accelerometer, 3D gyroscope and $3 \mathrm{D}$ magnetometer

- 1 integrated barometer for improved height estimation in areas with limited satellite visibility

- 1 integrated processor for precise position and attitude determination with tightly coupled sensor fusion

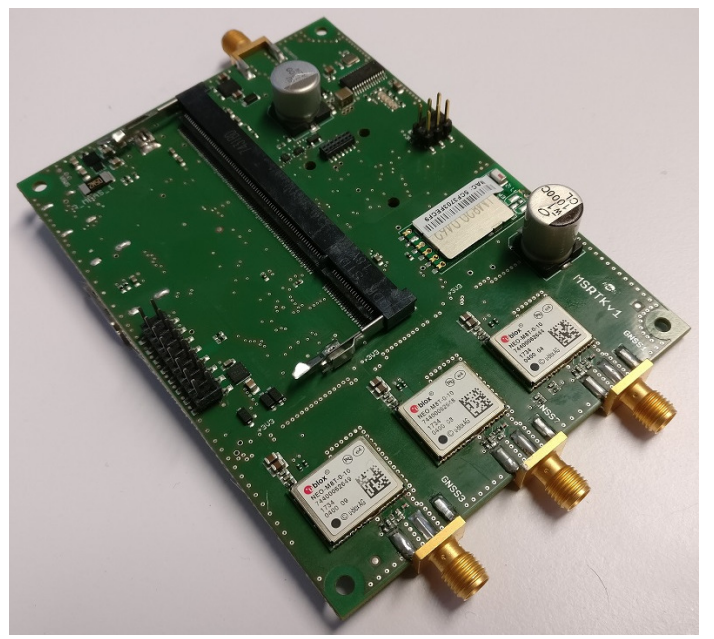

Figure 1. Multi-Sensor RTK Positioning Module of ANavS 


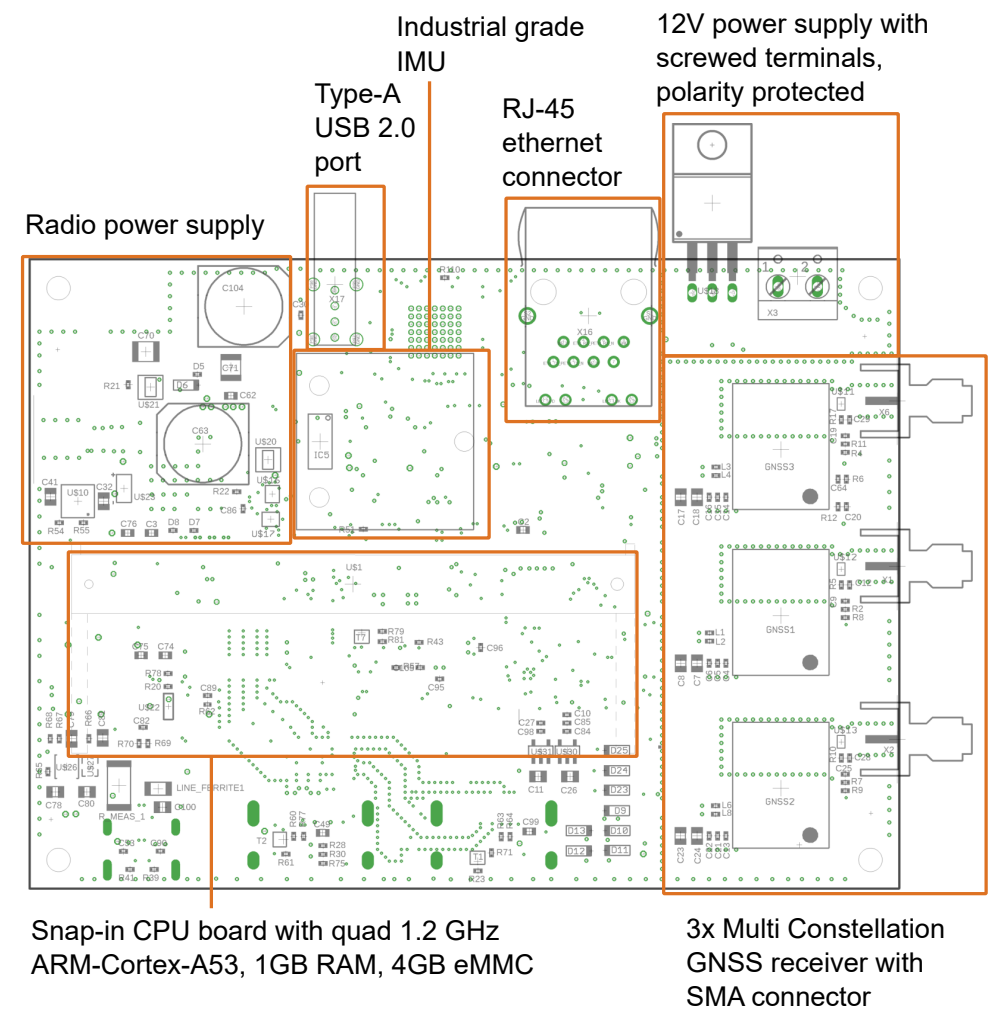

(a) top side

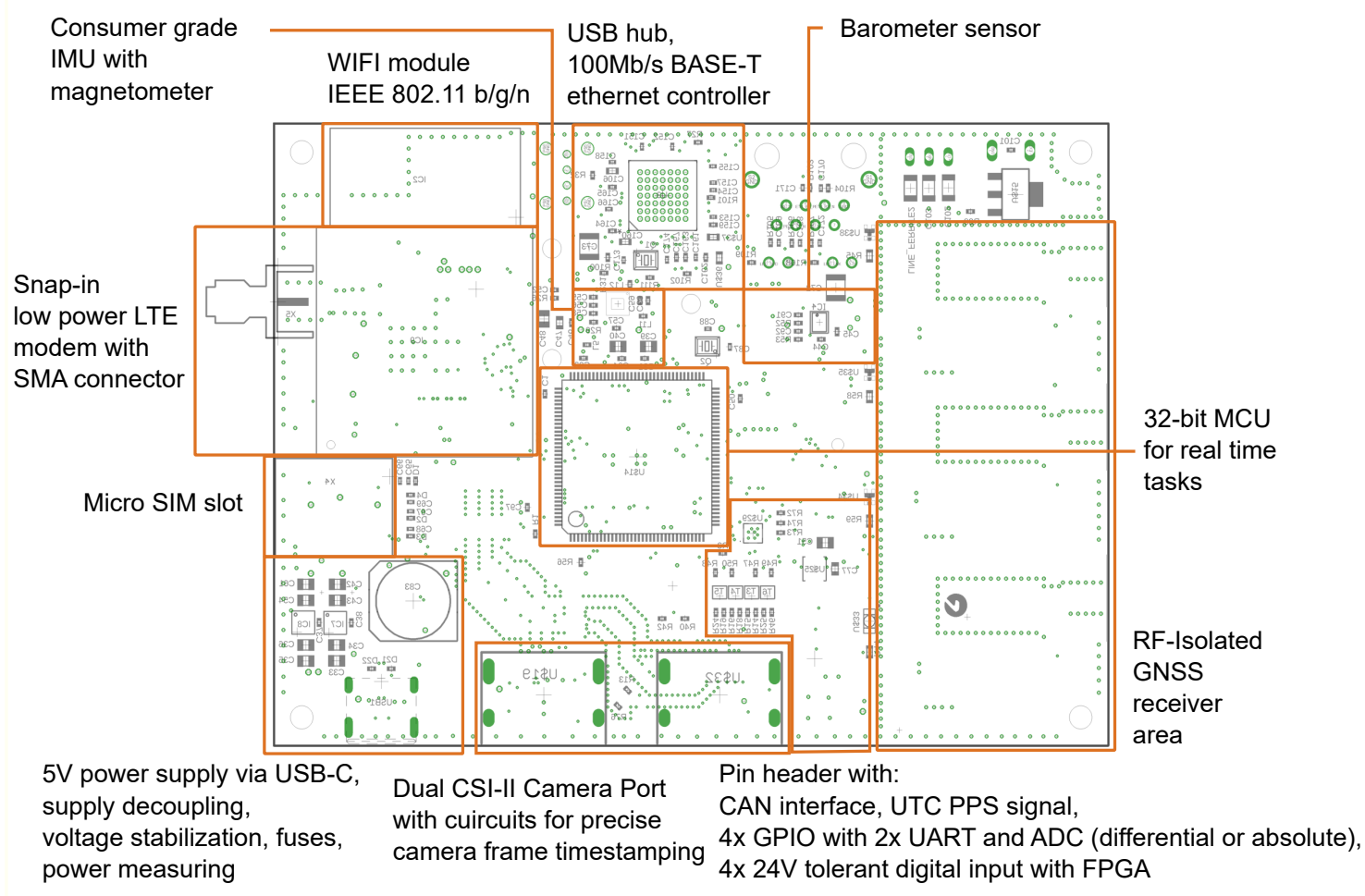

(b) bottom side

Figure 2. Multi-Sensor RTK Positioning Module 
- 1 integrated dual CSI-II camera port with circuits for precise camera frame time stamping

- 1 integrated LTE module for wireless reception of RTK corrections over the internet

- multiple communication interfaces: LTE, WLAN, Ethernet, CAN, UART, USB

Fig. 1 provides a picture of the ANavS Multi-Sensor, MultiGNSS RTK positioning module. The module has a very small form factor of only $110 \mathrm{~mm}$ x $80 \mathrm{~mm}$. Fig. 2 provides a more detailed picture of the board, i.e. the individual components and their placement on the top and bottom side of the board are shown.

\section{Performance of Multi-GnSS RTK Positioning}

In this section, we describe the performance of the proposed Multi-GNSS RTK positioning method with the new ANavS Multi-Sensor, Multi-GNSS RTK positioning module. Two ANavS modules were placed next to each other at Pinakothek in Munich, Germany, at a latitude of $\varphi=48.148841^{\circ}$ and a longitude of $\lambda=11.570333^{\circ}$ on May 25, 2018.

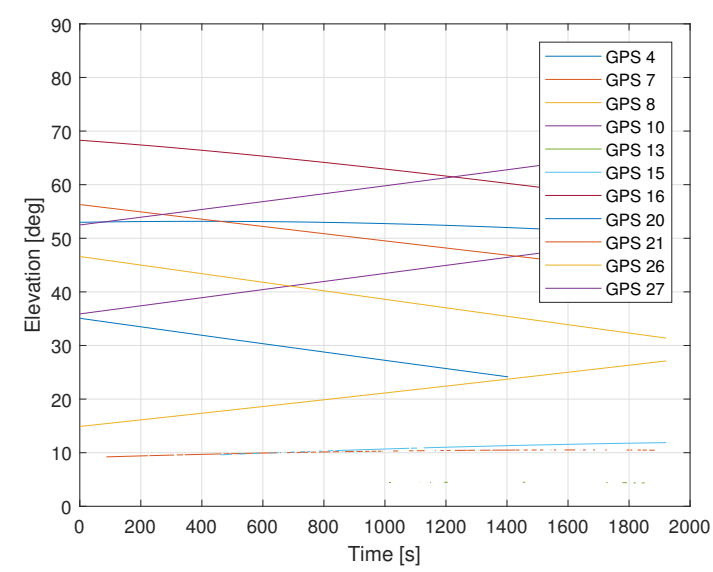

(a) GPS satellites

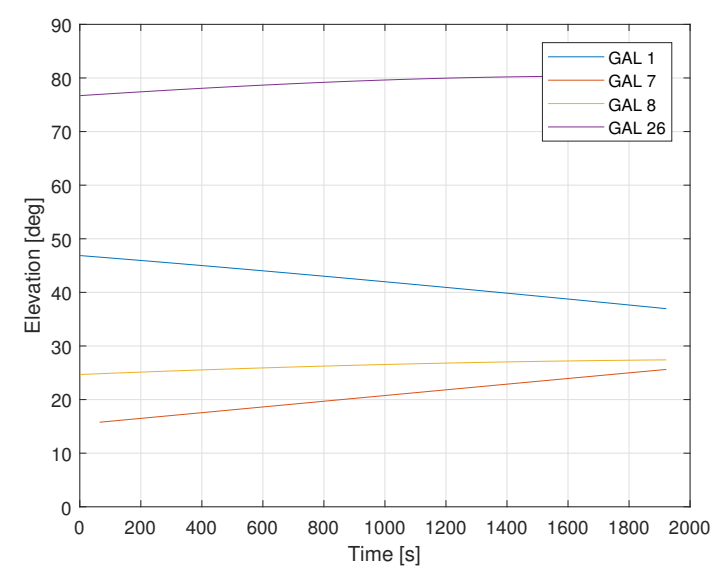

(b) Galileo satellites

Figure 3. Elevation of GNSS satellites during measurement at Pinakothek, Munich, Germany on May 25, 2018.

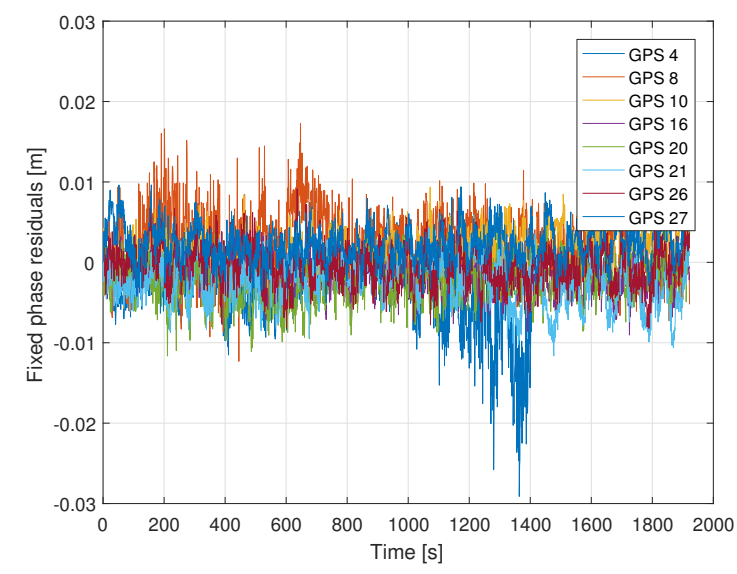

(a) GPS satellites

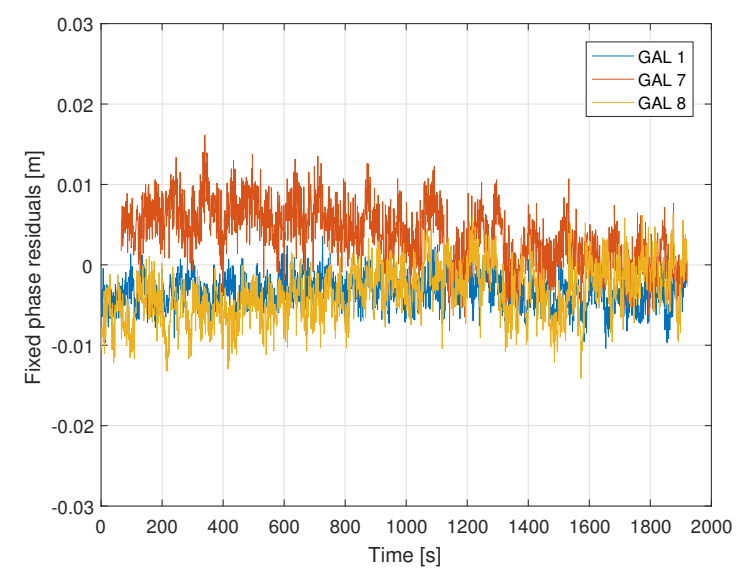

(b) Galileo satellites

Figure 4. Fixed phase residuals of GNSS double difference measurements at Pinakothek, Munich, Germany.

\section{A. Positioning Accuracy}

Fig. 3 shows the elevations of the visible GPS and Galileo satellites during the measurement of approximately $30 \mathrm{~min}$ utes. The Galileo satellite with PRN 26 had the highest elevation throughout the measurement. Therefore, it was chosen as common reference satellite for performing the double differences with all other visible GPS and Galileo satellites.

Fig. 4 shows the fixed phase residuals for the GPS satellites in subfigure a) and for the Galileo satellites in subfigure b). The residuals are below $1 \mathrm{~cm}$ for almost all satellites and epochs, which indicates a correct integer ambiguity fixing. The GPS satellite with PRN 4 is setting after $1400 \mathrm{~s}$ and the Galileo satellite with PRN 7 is raising just after $65 \mathrm{~s}$. Both satellites have a very low elevation and, therefore, are affected by substantial phase multipath resulting in increased phase residuals of up to $3 \mathrm{~cm}$.

Fig. 5 shows the horizontal relative position estimates between both RTK modules. We splitted the measurement data into three parts to test the repeatability with three independent ambiguity fixings. The figure shows the single-epoch least- 


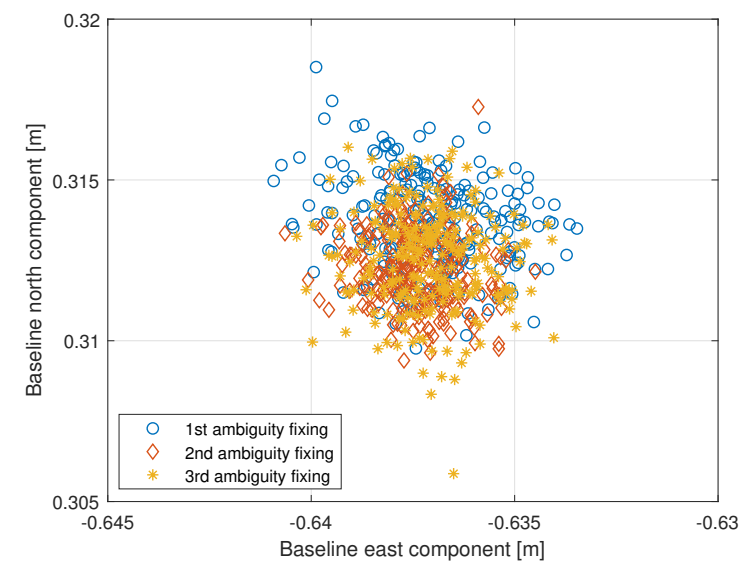

Figure 5. Single epoch least-squares baseline estimates for three different ambiguity fixings during measurement at Pinakothek, Munich.

squares baseline estimates as provided by Eq. (25) for the three ambiguity fixings. The position estimates vary within only a few millimeters and are consistent over the fixings.

\section{B. Sensitivity to Interference from LTE}

The GNSS measurements of the reference station are transmitted to the mobile user by LTE to enable differential positioning. The LTE signal is several orders of magnitude stronger than the GNSS signals, and some harmonics of the LTE signals are overlapping with the GNSS frequencies. Therefore, a careful design of the RTK module is required.

The ANavS Multi-Sensor, Multi-GNSS RTK module uses a shielded LTE module and external GNSS and LTE antennas to minimize the potential interference between LTE and GNSS. The interference of GNSS by LTE is analyzed by comparing the GNSS signal strength with active and powered down LTE module. Fig. 6 shows the carrier to noise power ratio of all visible GPS and Galileo satellites with powered down LTE module, and Fig. 7 refers to the signal strength with simultaneous LTE transmission.

The two tests were performed after each other, i.e. the satellite geometry has slightly changed between both tests. We can observe that the maximum carrier to noise power ratio is 1 to $3 \mathrm{~dB}$ lower during active LTE transmission, which is caused by some extent due to the LTE interference and to some extent by the change of the satellite geometry. Obviously, the LTEbased interference is quite small compared to the variation in signal strength between different satellites. The latter one is caused by the low-cost patch antennas, which have a typical $C / N_{0}$ degradation of $\geq 10 \mathrm{~dB}$ for low-elevation satellites compared to high-elevation satellites.

\section{CONClusion}

The bottleneck of any precise GNSS-based positioning is the carrier phase ambiguity fixing due the small wavelength. This paper provides a Real-Time Kinematic (RTK) positioning method, that uses the satellites from both GPS and Galileo, selects a common reference satellite, estimates pseudorange

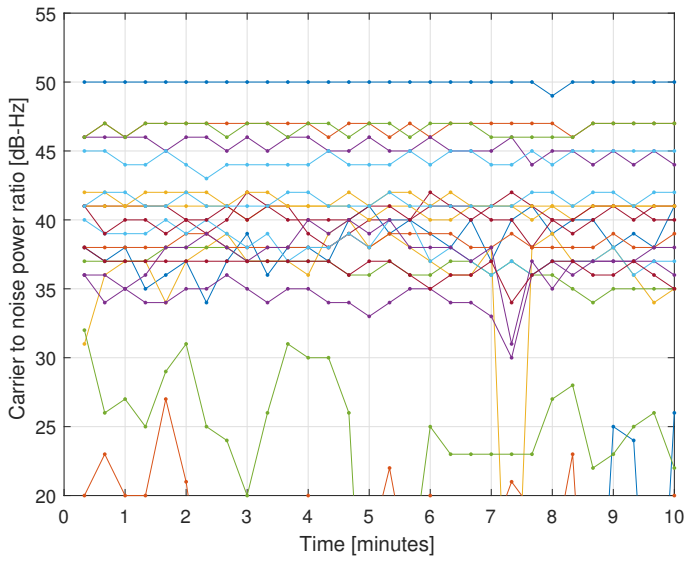

Figure 6. Carrier to noise power ratio of GNSS satellites with powered down LTE module.

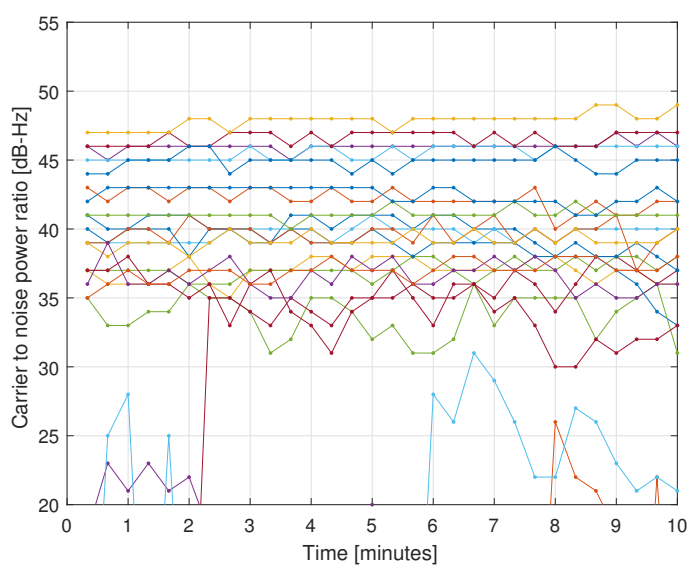

Figure 7. Carrier to noise power ratio of GNSS satellites with simultaneous transmission of LTE and GNSS signals.

multipath errors, and extends the LAMBDA method by hypothesis testing to overcome the previous shortcomings.

The method was implemented on the new ANavS MultiSensor, Multi-GNSS RTK positioning module, and provided a repeatable millimeter-level positioning accuracy. The interference of LTE signals on GNSS was also analyzed, and the measurement results show that the LTE-based interference is well-below the natural variation of the carrier to noise power ratio between different satellites.

\section{REFERENCES}

[1] P. J. Teunissen, "The least-squares ambiguity decorrelation adjustment: a method for fast GPS integer ambiguity estimation," Journal of Geodesy, vol. 70, pp. 65-82, 1995.

[2] P. Henkel, M. Iafrancesco, and A. Sperl, "Precise point positioning with multipath estimation," in Proc. of Position, Location and Navigation Symp. (PLANS), pp. 144-149, IEEE, 2016.

[3] P. Misra and P. Enge, "Global Positioning System: signals, measurements and performance second edition," Ganga-Jamuna Press, 2006.

[4] J. H. Hahn and E. D. Powers, "Implementation of the GPS to Galileo time offset (GGTO)," in Proc. of the Frequency Control Symposium and Exposition, pp. 33-37, IEEE, 2005.

[5] R. G. Brown and P. Y. Hwang, Introduction to random signals and applied Kalman filtering: with MATLAB exercises. John Wiley, 2012. 